\title{
A pilot randomised controlled trial of an Internet-based cognitive behavioural therapy self-management programme (MS Invigor8) for multiple sclerosis fatigue.
}

\author{
Rona Moss-Morris ${ }^{1 *}$, Paul McCrone ${ }^{2}$, Lucy Yardley ${ }^{1}$, Kirsten van Kessel ${ }^{3}$, Gary B Wills ${ }^{4}$, \\ Laura K.Dennison ${ }^{1}$
}

\author{
${ }^{1}$ School of Psychology, University of Southampton, Southampton, UK
}
${ }^{2}$ Centre for the Economics of Mental Health, Institute of Psychiatry, King's College London, London, UK

${ }^{3}$ Department of Psychology, University of Auckland, Auckland, New Zealand

${ }^{4}$ School of Electronics and Computer Science, University of Southampton, Southampton, UK

*Address for correspondence and reprints: Professor Rona Moss-Morris, School of Psychology, University of Southampton, Highfield Southampton, SO17 1 BJ, UK, email: remm@soton..ac.uk, telephone ++4402380 597549

Key words: multiple sclerosis, fatigue, cognitive behavioural therapy (CBT), Internetbased, self-management

Word count 3538 


\begin{abstract}
The majority of people affected by Multiple Sclerosis (PaMS) experience severe and disabling fatigue. MS Fatigue is poorly understood and most existing treatments have limited effectiveness. However, a recent randomised controlled trial (RCT) showed that cognitivebehaviour therapy with a clinical psychologist was effective in reducing MS fatigue severity and impact. The current study developed an Internet-based version of this intervention to make it available to a wider group of PaMS and conducted preliminary investigations of its efficacy, feasibility and cost-effectiveness in a pilot RCT.
\end{abstract}

The 'MS Invigor8' website was developed using agile design and substantial input from PaMS. The programme includes eight online tailored and interactive sessions along with homework tasks, intended to be accessed weekly. In the pilot trial, 40 patients were randomised to MS Invigor8 $(n=23)$ or standard care $(n=17)$. The MS Invigor8 group accessed sessions over 8-10 weeks and received up to three 30-50 minute telephone support sessions. Participants completed online questionnaires assessing fatigue, mood and quality of life at baseline and 10 weeks follow-up.

Large between group treatment effects were found for the primary outcomes of fatigue severity $(\mathrm{d}=1.19)$ and impact $(\mathrm{d}=1.22)$. The MS Invigor8 group also reported significantly greater improvements in anxiety and depression. Analysis suggested that the intervention may be cost-effective. Qualitative feedback suggested that participants considered this treatment approach acceptable and helpful. Technical website problems negatively affected some users' experiences and need to be resolved. Given the promising results a larger RCT with longer term follow-up is warranted. 
Multiple Sclerosis (MS) is a chronic, incurable neurological disease which affects more than 2.5 million people worldwide (1). Fatigue is reported by around $90 \%$ of people affected by MS (PaMS) with over two-thirds complaining it is their most troubling symptom (2). Fatigue is a major reason for unemployment in MS (3) and is associated with depression and inability to carry out day-to-day tasks $(4 ; 5)$.

Few treatments have been shown to be effective for MS fatigue. Evidence from pharmacotherapy trials is inconsistent and effects are modest at best $(4 ; 6)$. Other studies have used a more behavioural approach using either exercise or energy conservation methods but again results are mixed (3). These interventions are limited by the absence of a clear conceptualisation of MS fatigue. We recently developed a cognitive-behavioural conceptualisation of MS fatigue integrating the findings across biological and psychosocial research (6). Based on this model, we developed a cognitive behavioural therapy (CBT) programme which was shown in a randomised controlled trial (RCT) to effectively reduce fatigue up to six month follow-up (7).

Despite these promising results, rolling out individual CBT for MS fatigue is likely to be problematic. Few MS services have access to a CBT therapist and attending therapy sessions may be difficult for many PaMS. The provision of CBT-based self-management programmes over the Internet is a rapidly evolving and potentially beneficial means of delivering treatment otherwise unsought or unobtainable (8). There is growing evidence that Internetbased interventions are an effective form of treatment for a variety of physical symptoms, including back pain, headache and tinnitus (9-11) particularly when supplemented by some telephone support (12).

The initial aim of the current study was to develop an Internet self-management package for MS fatigue (MS Invigor8) based on our therapist-delivered CBT programme and formalised feedback from (PaMS). The second aim was to conduct a pilot RCT of MS Invigor8. The aims of the pilot were to 1) assess the feasibility of conducting automated trial randomisation and baseline and follow-up assessment procedures online, 2) evaluate adherence to the treatment programme, 3) assess the potential efficacy of the programme in reducing fatigue severity and impact (primary outcomes), and depressed and anxious mood (secondary outcomes) at 10 weeks post randomisation, 4) conduct a preliminary cost-effective analysis 
of the programme, and 5) gather detailed qualitative feedback on the programme from participants to make revisions if necessary before we consider a full sized RCT.

\section{Method}

\section{Phase 1: Developing MS Invigor8: Breaking the Cycle of Fatigue}

Agile design (13) was used to develop MS Invigor8. Agile design incorporates a co-design team who work closely together to produce software, whilst maintaining constant links with the end user. The design team included a software project manager (GW), software developers, health psychologists (RM, LY) and an expert paMS. The website was designed in a modular, iterative fashion. Six volunteers with MS fatigue (two men, four women, mean age 45, with different types and severity of MS, and differing computer skills) assisted the co-design team. They tested the modules in their own homes as they were developed. Immediately after completing each module, they were interviewed on the telephone using a variant of the 'think aloud' method (14) which is used to understand the different perspectives of individual users. Feedback was elicited about their experience of the module, focusing on issues of usability, usefulness and acceptability of the information, and suggestions for improvements. Interviews were recorded, transcribed verbatim, and analysed. The feedback was used to modify session content and programme functionality. The programme is summarised in table 1 and described under treatment below.

\section{Phase 2: Pilot Study}

\section{Design and trial procedures.}

The pilot RCT study was approved by the University of Southampton ethics committee. The website was designed to incorporate all trial and treatment procedures. Participant information sheets including eligibility criteria were presented online with the facility to contact the investigators for more information. PaMS who wanted to participate consented online and completed an online screening questionnaire to confirm eligibility. Those who were eligible could then consent to enter the trial and complete the baseline questionnaire. They were allocated to MSInvigor8 or a control group by an automated simple randomisation system built into the website. Participants were alerted to complete the online follow-up questionnaire at 10 weeks post randomisation. Control participants were given access to 
MSInvigor8 once they had completed the 10 week questionnaire, but they did not receive the telephone support.

\section{Recruitment and eligibility}

Recruitment was through advertisements on the MS Society and MS Trust websites and a local press release. Participants were required to be UK Resident, have a definite diagnosis of MS from a neurologist, have significant fatigue indicated by a score $>4$ on the Fatigue scale using the binary scoring method (15), and be ambulatory with or without a stick for at least 100 meters. Participants had to be willing to abstain from starting new treatments for fatigue for the study duration.

The recruitment target was to randomise 40 participants within the two month recruitment period. A pilot Phase II trial of at least 30 subjects is considered adequate for obtaining reasonably reliable sample size estimates (16). A slightly larger sample size allowed us to account for possible loss to follow-up to estimate sample size for a full Phase III trial as well as to assess trial methodologies and procedures across a range of people.

112 people underwent the automated screening process. 74 of the 112 screened did not register for the trial. Reasons for non-registration are outlined in Figure 1.

48 of the 112 people screened registered for the trial of whom 8 had to be withdrawn. 3 people were excluded as they were subsequently found to be non-UK resident. Five participants who registered and were randomised to the control group were withdrawn because a website bug allowed them to access sessions on MSInvigor8 during the control period. Twenty-three people were randomly allocated to the MSInvigor8 group and 17 to the control group. Recruitment took a total of 61 days.

\section{Treatment}

The programme entitled 'MS Invigor8: Breaking the Cycle of Fatigue' consists of 8 weekly sessions summarised in table 1 . On average, sessions take between 25 to 50 minutes to complete. All sessions are interactive and include self-assessments which allow the programme to be tailored to the individual problems and progress of the PaMS. Educational information can be printed. Sessions include homework tasks which are saved online in a 
Workbook, can be printed, and are reviewed in the subsequent session. Other features of the site include programme instructions, a 'take a break' button allowing people to rest during sessions, a time bar indicating progress through a session, and optional video/audio clips demonstrating relaxation techniques.

During the trial, the MS Invigor8 group received automated emails encouraging the completion of one session per week over 8-10 weeks. Participants also received three telephone support sessions of between 30-50 minutes, while they worked through the programme. The first was scheduled after the completion of the initial session and focused on talking through the personal five part model (see table 1, session 1). The second took place in week three and focused on clarifying goal setting and progress with goals. The final session in week six focused on identifying and challenging unhelpful thoughts. Sessions were provided by an assistant psychologist who received 5 hours of basic training in the interventions and fortnightly supervision from a registered psychologist (RMM). All sessions were audiotaped and fidelity to the treatment procedures was cross-checked throughout the trial.

\section{Assessments}

Participants provided demographic data, and information on their MS type and duration. Questions to quantify MS type were drawn from previous research (17). Ambulation ability was measured using the ambulation questions from the self-report Expanded Disability Status Scale (18).

The primary outcomes were:

1) Fatigue severity, measured by the ordinal version of the Fatigue Scale (15). This questionnaire measures physical and mental fatigue severity, was used in the CBT therapist trial for MS fatigue (7) and has good psychometric properties . Cronbach's $\alpha$ in this sample was excellent (.83)

2) Fatigue impact, assessed by Modified Fatigue Impact Scale (19), a shortened version of the Fatigue Impact Scale (20). This scale taps perceived limitations in cognitive, physical and psychosocial functioning as a result of fatigue. The scale has been validated in people with MS (20). Cronbach's $\alpha$ in this sample was excellent (.81) 
The secondary outcomes were anxiety and depression, measured by the Hospital Anxiety and Depression scale (HADS) which was specifically designed to measure mood disturbance in people with physical illness (21). Cronbach's $\alpha$ was .84 for anxiety and .72 for depression.

For the economic evaluation, participants completed the EuroQol (EQ5D) (22) as a measure of quality-adjusted life years (QALYs), and service use during the 10-week follow-up period was measured with an adapted version of the Client Service Receipt Inventory (CSRI) (23).

Fifteen of the 23 MS Invigor8 participants agreed to be interviewed about their experiences of the intervention. A health psychology researcher independent of the trial team conducted semi-structured interviews based around experiences of fatigue, treatment expectations and how the participant was feeling following the intervention. Participants were also asked about experiences of using MS Invigor8 and the telephone support and specific elements they had found helpful and unhelpful. Data-driven thematic analysis was used to identify common and salient themes $(24 ; 25)$.

\section{Statistical analysis}

Analyses were performed using SPSS for Windows (Version 17; SPSS Inc, Chicago, IL). As this is the pilot study, data were presented descriptively. We also conducted treatment effect analyses by intention-to-treat; for participants that dropped out, baseline scores were carried forward to post-treatment. The exception to this was the five control participants who had erroneously accessed MSInvigor8. They only provided baseline data and were removed entirely from all analyses as they were deemed to no longer be true controls. As they did not provide follow-up data, their inclusion would have seriously biased the results in favour of the treatment group. Differences between groups were analysed using general linear models with group as the fixed factor, baseline values as covariates and post treatment scores as the dependent variable.

\section{Economic analysis}

Service costs were calculated by combining the service use data with appropriate unit costs (26). QALYs were calculated by adding the baseline and follow-up EQ-5D scores and dividing by 2 , assuming a linear (change over time) and multiplying by $10 / 52$ (which is the maximum QALY gain attainable in the follow-up period). 


\section{Results}

\section{Participants}

Table 2 shows characteristics of the participants. The mean age was early forties, most participants were female, and 30\% were unemployed or working less because of their MS. The groups were well matched for age and time since diagnosis. However, the intervention group had a slightly lower percentage of females, a greater percentage of people with progressive disease, and greater levels of ambulatory difficulties than the control group.

Three of the 23 treatment participants failed to complete follow-up assessments. They were the lowest users of the site, with one not completing any sessions. One of the controls did not complete follow-up assessment but no reason was given.

\section{Adherence to intervention}

At the 10 week assessment point the mean number of sessions completed was 4.91 (SD 2.10). Only one (4.3\%) participant finished all 8 sessions and three (13\%) finished 7 sessions. Overall $15(60.8 \%)$ patients finished more than half the sessions (>5) with only one $(4.3 \%)$ patient not completing any sessions.

Participants still had access to the website after the end of the assessment period and continued to complete sessions as captured by the automated record of sessions completed. The mean number of sessions completed one month post the assessment period was 6.1 (SD 2.45) with 11 people having completed all 8 sessions (47.8\%) and 19 (82.5\%) having completed more than half the sessions.

21 (91\%) participants completed the initial telephone support session (mean length 52.43 minutes, SD 15.35). 19 (82\%) participants completed the second session (mean length 30.21 minutes, SD 7.8) and 15 (65\%) completed the final session (mean length 45.6 minutes, SD 14.18). Reasons for cancellations included work commitments or other appointments, illness, not having completed the MS Invigor8 session and non-response. No adverse events were reported.

\section{Descriptive outcome data}


The means and standard deviations for the outcome data across groups are presented in table 3. The groups appear well matched on baseline levels of fatigue and mood. The control group appears to show little change over the 10 weeks in all four outcomes, whilst the treatment group shows decreases on both fatigue measures as well as anxiety and depression.

\section{Analysis of Primary outcomes}

The MS Invigor8 group showed significant reductions when compared to the control group on both the Fatigue Scale $(F(2,37)=15.55, p<.001)$ and the Modified Fatigue Impact Scale $(F(2,37)=14.67, p<.001)$. The between group effect size for the fatigue scale was $\mathrm{d}=1.19$ [95\% CI -1.28-3.98] and for the Modified Fatigue Impact Scale, d=1.22 [95\% CI -0.62-2.31].

\section{Analysis of Secondary outcomes}

There were significant differences between the groups on anxiety $(F(2,36=12.31, p=.001)$ and depression $(F(2,36)=13.33, p=.001)$, with the MS Invigor8 group showing significantly greater reductions on both scales.

We re-ran the outcome analysis controlling for gender and ambulation status and a completer only analysis (those who returned follow-up), and the results retained their significance levels for all outcomes.

\section{Cost-effectiveness}

During the follow-up period the most commonly used services were neurologists and general practitioners (Table 4). Mean costs were very similar in the intervention (£211) and control (£214) groups. There is therefore no evidence that during the treatment period the intervention had any impact on the use and cost of other services. Medication costs were not included, although it is unlikely these would differ between groups. Two participants in the control group did start to receive a disease modifying agent during the follow-up period but it is difficult to assess whether this would be due to lack of the intervention.

The mean (SD) EQ-5D scores at baseline were $0.56(0.26)$ in the intervention group and 0.64 (0.28) in the control group. By follow-up the scores were $0.71(0.28)$ in the intervention group and $0.62(0.27)$ in the control group. The mean score at follow-up, adjusted for baseline, was 0.15 higher for the intervention group ( $\mathrm{p}=0.038)$. The mean (SD) QALY gain 
for the intervention group was 0.1212 and 0.1243 in the control group. However, due to the quality of life difference at baseline the QALY gain was 0.015 higher in the intervention group $(\mathrm{p}=0.038)$.

\section{Qualitative feedback from people in the treatment group.}

The interviews $(n=15)$ revealed that most participants perceived an improvement in their fatigue, felt they understood their fatigue better, were more in control of it and were trying to implement lifestyle changes suggested by the programme.

"It's like somebody offering a helping hand, and that psychologically has been a huge boost to me. That there is this therapy out there that can make a difference. It's not going to cure my MS, but it will help me handle it." (female, 60 years)

"I wasn't aware of how much certain things did have an impact on my fatigue, and how I could go around and help myself by sort of training my brain to think differently almost, um, or to deal with things differently," (females, 36 years)

"I don't have any fatigue at the moment um compared to when I started I feel more have more higher energy levels" (male, 32 years)

The telephone support was perceived as augmenting the Internet-based sessions by nearly all participants.

"It was actually really good to talk to her because I was able to relate what I was learning ... in the sessions, to my personal circumstances." (female, 35 years)

"The programme on its own, without the therapy sessions, would be .. 50 percent as effective. The talking ... sessions, certainly for me, have been vital." (female, 60 years)

The structure of the programme was generally well accepted but occasional technical difficulties with the programme were detrimental to many users' experiences, especially those using certain Internet browsers. Participants also offered suggestions for modifications to website layout and operation. Further customisation was seen as necessary by some, 
including a few people with progressive forms of MS suggesting that the programme may be more relevant to those with relapsing-remitting MS.

\section{Discussion}

This study developed a novel Internet based intervention, MS Invigor8, which has the potential to make CBT based self-management for fatigue more widely available to PaMS. Strengths of the programme included its iterative development process with input from PaMS and its basis on a theoretical and evidenced-based approach for treating MS fatigue $(6 ; 7)$. The pilot data suggest that MSInvigor8 has the potential to effectively reduce both severity of fatigue and its impact on daily living. The between group effect sizes for the fatigue measures at the end of treatment were well above the 0.80 cut-off for a large treatment effect (27). MS Invigor8 also had a significant positive effect on both anxiety and depression. These data add to a growing body of literature showing that Internet-delivered self-help CBT with minimal therapist support is an effective treatment option for a range of symptom based problems such as tinnitus, headache, chronic pain, insomnia and irritable bowel syndrome $(28 ; 29)$.

In terms of economics, we measured other service costs rather than direct cost of the intervention because the latter would depend on how many people in routine practice made use of it. Service costs were very similar between the groups over the 10 week period. However, this period may be too short to show much change in service use costs. The intervention produced more QALYs. To achieve a cost per QALY of $£ 20,000$ (below which interventions in England are usually recommended) the intervention costs would need to be no more than $£ 300$ per person ( $£ 20000$ divided by 0.015 QALYs), or approximately $£ 50$ per session. If the intervention were never used beyond the 23 in the intervention group then this would only cover $£ 6900$ development costs. However, if the intervention is effective it is likely to be used by many more. If 300 people used it then this would cover a $£ 90,000$ development cost which is above the actual costs that were accrued. More widespread use would clearly offset these costs to greater extent, as would maintaining the QALY gain beyond 10 weeks. 
Internet delivery of the intervention appeared to be acceptable to most participants. PaMS self-referred into this study. Recruitment targets were met quickly suggesting that PaMS are self motivated to receive such treatments. There were also few drop outs. In depth interview feedback was largely positive, with many participants finding MS Invigor8 more helpful than other treatments they had tried. The telephone support was also seen by most as a key component of the treatment. Negative feedback centered on problems with website bugs rather than the nature of the treatment. Additional feedback was given to improve on minor aspects of the intervention structure and content. These revisions and bug fixing will improve the programme for future users.

The adherence data suggests that patients struggled to complete the programme within ten weeks. Interestingly a number of people continued with the website treatment after the study was finished. Thus, a longer treatment period may be required, possibly with more structured support by email or telephone to assist with progress.

In the original therapist trial of CBT for MS fatigue, all PaMS in the CBT group completed the 8 sessions (7). The average change on the Fatigue Scale in the original trial was 13.04 points compared to 9 points in the current study. The eligibility criteria for both trials were the same and the baseline fatigue scores were similar. It would therefore be interesting to investigate the relative clinical and cost-effectiveness of these two approaches in a future trial. Further research comparing different doses of therapist time alongside the website would help ascertain optimal contact time. Therapist skill may also influence treatment effects; therapy support in this study was provided by a psychology assistant with minimal CBT experience, whereas the therapy trial included an experienced clinical psychologist

This study suffered certain limitations linked to the Internet technology. The development of the website proved extremely time-consuming. This part of the project overran leaving insufficient time to adequately test the website before we went to trial. This meant that many participants encountered bugs in the programme. Most could work around these, but the qualitative data suggested that the problems negatively affected participants' treatment experiences. Problems also occurred with using the website for trial procedures such as screening and self-entry into the trial. It had been hoped that incorporating these into the website would be a feasible means of reducing trial research costs and widening the 
recruitment base. However, our experiences suggest that handling these trial procedures independently will provide better control over recruitment and eligibility.

The current study was a small pilot/feasibility trial of volunteers recruited over the internet and there was no long term follow-up data. A larger more definitive RCT is now needed with longer term follow up to examine maintenance of treatment gains.

\section{Conclusion}

Internet CBT based self-management appears to be a promising, acceptable and cost-effective approach for treating MS fatigue and improving broader outcomes such as distress. If future research replicates these findings MSInvigor8 may be a feasible means of delivering a CBTbased intervention to a large pool of PaMS who experience troubling and disabling fatigue. 


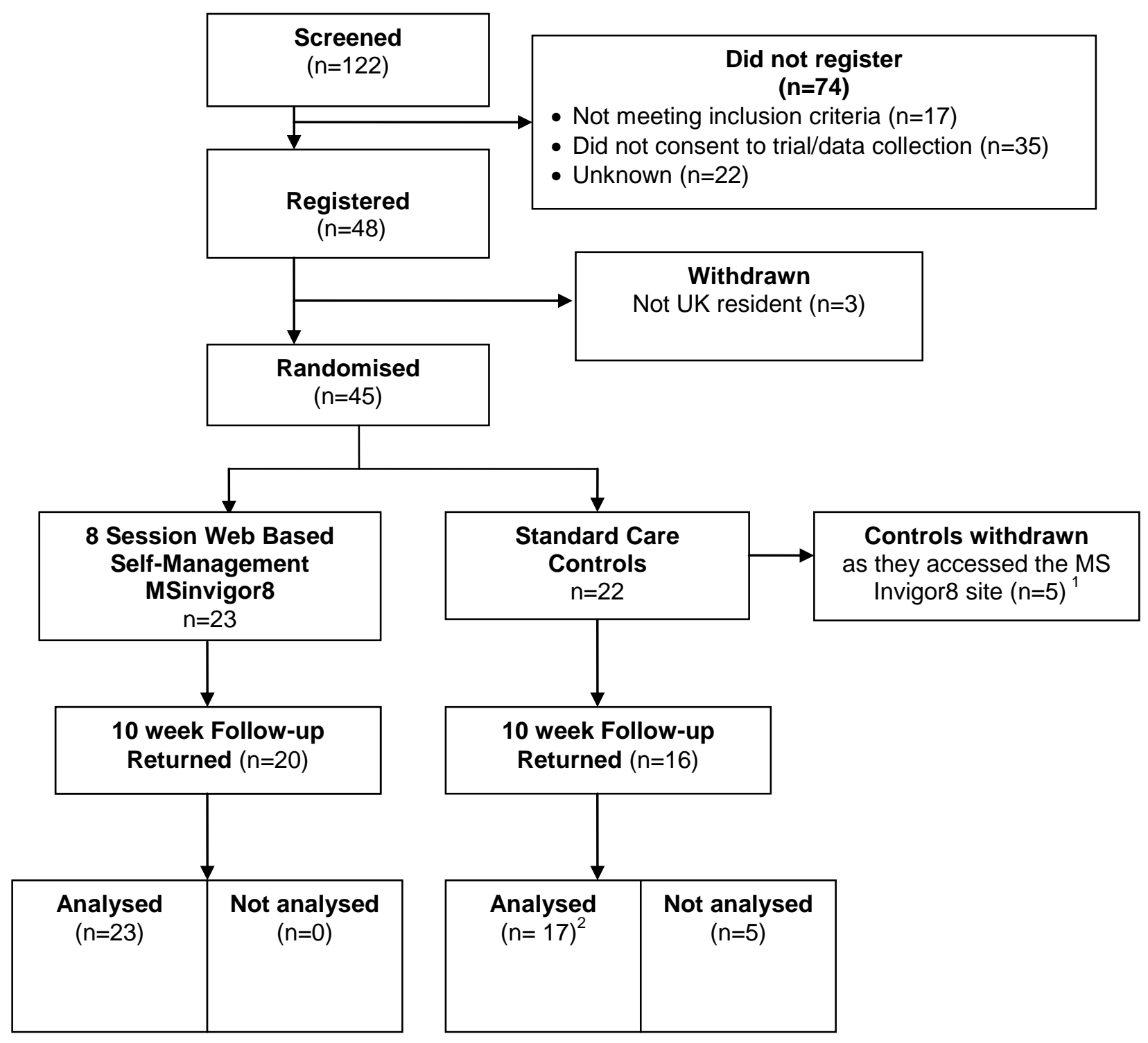

Figure 1: participant flow through the trial

${ }^{1}$ An initial website fault meant that 5 participants allocated to the waiting list control were able to access the web site. These people were withdrawn from the trial

${ }^{2}$ Baseline data missing for one control on three of the outcome measures. For these analyses only 16 controls are included but the primary data is analysed for 17 
Table 1: Outline of MSInvigor8 sessions.

\begin{tabular}{|c|c|}
\hline Session & ontent \\
\hline 1 & $\begin{array}{l}\text { Title: Understanding MS fatigue } \\
\text { Overview of MS fatigue. Self-assessment of biological, behavioural, cognitive, emotional and } \\
\text { environmental factors which may be contributing to fatigue. Computer generated 5-part personal } \\
\text { cognitive behavioural (CB) model of MS fatigue based on self-assessment data. }\end{array}$ \\
\hline 2 & $\begin{array}{l}\text { Title: Fatigue Diary } \\
\text { Introduction to self-management based on CBT principles including the role of homework. } \\
\text { Interactive tasks include the steps of keeping daily diaries of activity, rest and fatigue levels. } \\
\text { Homework: Daily fatigue diary }\end{array}$ \\
\hline 3 & $\begin{array}{l}\text { Title: Rest and Activity Patterns. } \\
\text { Review of diary. Education on how erratic patterns of rest and activity or over-activity affect } \\
\text { fatigue. Importance of consistency is outlined, and benefits of moderate physical exercise } \\
\text { discussed. Interactive tasks include the steps of goal setting (what, when, where). } \\
\text { Homework: Set and monitor rest and activity goals and exercise goals }\end{array}$ \\
\hline 4 & $\begin{array}{l}\text { Title: Improving Sleep } \\
\text { Self-assessment of sleep patterns. Education on behavioural techniques (basic sleep hygiene) to } \\
\text { improve sleep are discussed, e.g. having set 'worry times', getting up if lying awake in bed for } \\
\text { longer than } 20 \text { minutes. } \\
\text { Homework: Set and monitor goals for improving sleep. }\end{array}$ \\
\hline 5 & $\begin{array}{l}\text { Title: Understanding MS Symptoms } \\
\text { Education on normal symptom fluctuations versus signs of relapse and disease progression. The } \\
\text { effect on fatigue of symptom focusing and attributing all symptoms to MS is introduced. } \\
\text { Interactive task to generate possible alterative attributions for somatic symptoms e.g. stress, } \\
\text { medication side effects. } \\
\text { Homework: Recording somatic symptoms and generating alternative attributions. }\end{array}$ \\
\hline 6 & $\begin{array}{l}\text { Title: Recording Thoughts } \\
\text { The concept of unhelpful thoughts is introduced, and how these impact on fatigue and mood. } \\
\text { Interactive tasks include identifying cognitive errors and generating alternate thoughts. } \\
\text { Homework: Daily thought records of unhelpful thoughts and possible alternatives. }\end{array}$ \\
\hline 7 & $\begin{array}{l}\text { Title: Managing Stress } \\
\text { Basic stress management is discussed. Interactive tasks include identifying stressors which are } \\
\text { controllable and those which are not, and matching these to appropriate stress management } \\
\text { strategies. } \\
\text { Homework: Goals for stress management }\end{array}$ \\
\hline 8 & $\begin{array}{l}\text { Title: Emotions, Support and the Future } \\
\text { The importance of social support is discussed, and how to access better social support. Interactive } \\
\text { tasks include identifying possible sources of support for different types of support needed. } \\
\text { Managing difficult emotions and relapse are reviewed. } \\
\text { Homework: Building tool set for long term fatigue management and possible relapse. }\end{array}$ \\
\hline
\end{tabular}


Table 2: Baseline demographic characteristics of MS participants in the CBT and Control Groups.

\section{MS Invigor8 group}

$(\mathbf{n}=\mathbf{2 3})$ $40.14(17.76)$

$21(9.05)$

(16) $69.6 \%$

$13.95(4.83)$

Control group $(\mathbf{n}=17)$

Age (M,SD)

Time since diagnosis in years (M,SD)

Gender (n) \% female

$13.95(4.83)$

Marital status (n) \%

-Single

-Living with partner

- Divorced/widowed

- Missing data
(4) $17.4 \%$
(4) $26.7 \%$
(14) $60.9 \%$
(8) $53.3 \%$
(5) $21.7 \%$
(3) $17.6 \%$
(2) $11.8 \%$

Ambulation status (n) \%

- Able to walk $500 \mathrm{~m}$ or more without aid

(9) $39.1 \%$

(10) $58.8 \%$ or rest

- Able to walk $100 \mathrm{~m}$ without aid or rest
(4) $17.4 \%$
(2) $11.8 \%$
(9) $39.1 \%$
(4) $23.5 \%$

- Require unilateral or bilateral aid to

walk 20 to $100 \mathrm{~m}$

- Missing data

(1) $4.4 \%$

(1). $5.9 \%$

MS type (n) \%

-Relapsing remitting
(10) $43.5 \%$
(7) $30.4 \%$
(12) $70.6 \%$
(2) $8.7 \%$
(2) $11.8 \%$
(4) $17.4 \%$
(3) $17.6 \%$

-Secondary progressive

-Unsure

Employment related to MS

-working less or off sick

(2) $9.0 \%$

(7) $31.8 \%$

(1) $5.8 \%$

-unemployed

(4) $23.5 \%$

Note. $\mathrm{M}=$ mean; $\mathrm{SD}=$ standard deviation. 
Table 3: Means and standard deviations for the primary and secondary outcome measures across groups at baseline and 10 weeks follow-up.

\begin{tabular}{lcc}
\hline Group & $\begin{array}{c}\text { Pre-treatment } \\
\text { (baseline) }\end{array}$ & $\begin{array}{c}\text { Post treatment } \\
(\mathbf{1 0} \text { weeks) }\end{array}$ \\
\hline Fatigue Scale, mean (SD) & $12.39(6.84)$ \\
MSInvigor8 (n=23) & $21.39(4.30)$ & $19.57(5.20)$ \\
Control group (n=17) & $21.53(3.62)$ & $9.00(3.75)$ \\
& Modified Fatigue Impact Scale, mean (SD) \\
MSInvigor8 (n=23) & $13.17(3.81)$ & $12.88(3.89)$ \\
Control group (n=17) & $12.69(3.89)$ & $6.44(3.91)$ \\
& HADS Anxiety, mean (SD) & $11.65(5.26)$ \\
MSInvigor8 (n=23) & $8.26(4.31)$ & \\
Control group (n=16) & $9.56(4.50)$ & $5.18(3.38)$ \\
& & $8.73(3.62)$ \\
MSInvigor8 (n=23) & $7.96(3.64)$ & \\
Control group (n=16) & $6.75(2.72)$ & \\
\hline
\end{tabular}


Table 4. Service use and costs (2007/8 £s) at follow-up.

\begin{tabular}{|c|c|c|c|c|c|c|}
\hline \multirow[b]{2}{*}{ Service } & \multicolumn{3}{|c|}{ MSInvigor8 } & \multicolumn{3}{|c|}{ Control } \\
\hline & $\begin{array}{c}\mathrm{N}(\%) \\
\text { using } \\
\text { service }\end{array}$ & $\begin{array}{c}\text { Mean } \\
\text { (SD) } \\
\text { contacts }^{1}\end{array}$ & $\begin{array}{c}\text { Mean } \\
\text { (SD) } \\
\text { cost }^{2}\end{array}$ & $\begin{array}{c}\text { N (\%) } \\
\text { using } \\
\text { service }\end{array}$ & $\begin{array}{c}\text { Mean } \\
\text { (SD) } \\
\text { contacts }^{1}\end{array}$ & $\begin{array}{c}\text { Mean } \\
\text { (SD) } \\
\text { cost }^{2}\end{array}$ \\
\hline Neurology outpatient & $3(15)$ & $1.7(1.2)$ & $32(92)$ & $6(38)$ & $1.2(0.4)$ & $56(81)$ \\
\hline Other outpatient & $3(15)$ & $1.0(0.0)$ & $15(36)$ & $3(19)$ & $1.7(1.2)$ & $31(79)$ \\
\hline Neurology day-patient & $1(5)$ & $1.0(-)$ & $6(26)$ & $0(0)$ & - & $0(0)$ \\
\hline Residential care & $0(0)$ & - & $0(0)$ & $0(0)$ & - & $0(0)$ \\
\hline Urology inpatient $^{3}$ & $0(0)$ & - & $0(0)$ & $0(0)$ & - & $0(0)$ \\
\hline Intensive care unit ${ }^{3}$ & $0(0)$ & - & $0(0)$ & $0(0)$ & - & $0(0)$ \\
\hline Other inpatient & $2(10)$ & $1.0(0.0)$ & $49(152)$ & $1(6)$ & $1.0(-)$ & $31(123)$ \\
\hline General practitioner & $11(55)$ & $1.3(0.5)$ & $24(29)$ & $11(69)$ & $3.9(8.3)$ & $28(25)$ \\
\hline Neurologist (non-outpatient) & $4(20)$ & $1.0(0.0)$ & $17(36)$ & $6(38)$ & $1.0(0.0)$ & $23(35)$ \\
\hline Other specialist & $0(0)$ & - & $0(0)$ & $0(0)$ & - & $0(0)$ \\
\hline Physiotherapist & $3(15)$ & $1.7(0.6)$ & $7(21)$ & $4(25)$ & $2.3(1.0)$ & $17(35)$ \\
\hline Social worker & $2(10)$ & $4.0(2.8)$ & $43(185)$ & $0(0)$ & - & $0(0)$ \\
\hline Nurse & $3(15)$ & $1.0(0.0)$ & $3(7)$ & $5(31)$ & $3.4(3.9)$ & $20(56)$ \\
\hline Home help & $1(5)$ & $4.0(-)$ & $13(57)$ & $0(0)$ & - & $0(0)$ \\
\hline Other & $3(15)$ & $2.0(1.0)$ & $2(7)$ & $2(13)$ & $1.0(0.0)$ & $2(4)$ \\
\hline Total & & & 211 (299) & & & 214 (239) \\
\hline
\end{tabular}

${ }^{1}$ Contacts are for those using services only, ${ }^{2}$ Costs are for all participants, ${ }^{3}$ Inpatient contacts refer to number of days. 


\section{Reference List}

(1) National MS Society. Who gets MS? http://www.nationalmssociety.org/aboutmultiple-sclerosis/who-gets-ms/index.aspx . 2009. 18-2-2009. Ref Type: Online Source

(2) Giovannoni G. Multiple sclerosis related fatigue. Journal of Neurology Neurosurgery and Psychiatry 2006;77(1):2-3.

(3) Krupp LB, Serafin DJ, Christodoulou C. Multiple sclerosis-associated fatigue. Expert Review of Neurotherapeutics 2010;10(9):1437-47.

(4) Branas P, Jordan R, Fry-Smith A, Burls A, Hyde C. Treatments for fatigue in multiple sclerosis: a rapid and systematic review. Health technology assessment (Winchester, England) 2000;4(27):1.

(5) Schwartz CE, Coulthard-Morris L, Zeng Q. Psychosocial correlates of fatigue in multiple sclerosis. Archives of Physical Medicine and Rehabilitation 1996;77(2):16570.

(6) van Kessel K, Moss-Morris R. Understanding multiple sclerosis fatigue: A synthesis of biological and psychological factors. Journal of Psychosomatic Research 2006;61(5):583-5.

(7) van Kessel K, Moss-Morris R, Willoughby E, Chalder T, Johnson M, Robinson E. A randomized controlled trial of cognitive behavior therapy for multiple sclerosis fatigue. Psychosom Med 2008;70:205-13.

(8) Griffiths KM, Christensen H. Review of randomised controlled trials of Internet interventions for mental disorders and related conditions. Clinical Psychologist 2006;10(1):16-29.

(9) Andersson G, Stromgren T, Strom L, Lyttkens L. Randomized controlled trial of Internet-based cognitive behavior therapy for distress associated with tinnitus. Psychosom Med 2002;64(5):810-6.

(10) Andersson G, Lundstrom P, Strom L. Internet-based treatment of headache: Does telephone contact add anything? Headache 2003;43(4):353-61.

(11) Zachriat C, Kroner-Herwig B. Treating chronic tinnitus: Comparison of cognitivebehavioural and habituation-based treatments. Cognitive Behaviour Therapy 2004;33(4):187-98.

(12) Buhrman M, Faltenhag S, Strom L, Andersson G. Controlled trial of Internet-based treatment with telephone support for chronic back pain. Pain 2004;111(3):368-77.

(13) Cockburn A, Highsmith J. Agile software development: The business of innovation. IEEE Computer 2001;34(9):120-7. 
(14) van den Haak MJ, de Jong MDT, Schellens PJ. Evaluation of an Informational Web Site: Three Variants of the Think-aloud Method Compared. Technical Communication 2007;54:58-71.

(15) Chalder T, Berelowitz G, Pawlikowska T, Watts L, Wessely S, Wright D, et al. Development of a fatigue scale. Journal Of Psychosomatic Research 1993;37(2):14753.

(16) Browne RH. On the use of a pilot sample for sample size determination. Statistics in Medicine 1995;14(17):1933-40.

(17) Skerrett TN, Moss-Morris R. Fatigue and social impairment in multiple sclerosis: The role of patients' cognitive and behavioral responses to their symptoms. Journal of Psychosomatic Research 2006;61:587-93.

(18) Bowen J, Gibbons L, Gianas A, Kraft G. Self-administered Expanded Disability Status Scale with functional system scores correlates well with physicianadministered test. Multiple Sclerosis 2001;7:201-6.

(19) Fatigue guidelines development panel of the multiple sclerosiscouncil for clinical practice guidelines. Fatigue guidelines development panel of the multiple sclerosis council for clinical practice guidelines: evidence-based management strategies for fatigue in multiple sclerosis. Washington DC; 1998.

(20) Fisk JD, Ritvo PG, Ross L, Haase DA, Marrie TJ, Schlech WF. Measuring the functional impact of fatigue- intitial validation of the fatigue impact scale. Clinical Infectious Diseases 1994;18:S79-S83.

(21) Zigmond AS, Snaith RP. The hospital anxiety and depression scale. Acta Psychiatr Scand 1983;Jun;67(6):361-70.

(22) Curtis L, Netten A. Unit costs of health and social care. Canterbury: PSSRU; 2006.

(23) Beecham J, Knapp M. Costing psychiatric interventions. Gaskell, London: 2001.

(24) Boyatzis RE. Transforming Qualitative Information. Thousand Oaks, CA: Sage Publications; 1998.

(25) Braun B, Clarke V. Using thematic analysis in psychology. Qualitative Research in Psychology 2006;3:77-101.

(26) Curtis L. Unit Costs of Health and Social care. Canterbury.: PSSRU; 2008.

(27) Cohen J. Statistical power for the behavioral sciences (2nd ed.). Hillsdale: NJ: Erlbaum.; 1988.

(28) Ljotsson B, Falk L, Vesterlund AW, Hedman E, Lindfors P, Ruck C, et al. Internetdelivered exposure and mindfulness based therapy for irritable bowel syndrome-A randomized controlled trial. Behaviour Research and Therapy 2010;48(6):531-9.

(29) Andersson G. Using the Internet to provide cognitive behaviour therapy. Behaviour Research and Therapy 2009 Mar;47(3):175-80. 


\section{Acknowledgements:}

This study was funded by a grant from the UK Multiple Sclerosis Society. We would like to acknowledge the substantial input into this project of our design team including the trial coordinator, Louise Bell, programmers - Prins Adeel Butt, Lisha Chen-Wilson, and Stephanie Cobb, and Lawrence Gilbert. We would also like to thank Caroline Blakemore who conducted the think aloud interviews for the development of the website and Carolyn Powell who conducted and analysed the qualitative interviews for the pilot trial.

There were no competing interests. 
